\title{
Hiperplasia nodular focal múltipla do fígado - subtipo misto hiperplásico/adenomatoso
}

\section{Multiple focal nodular hyperplasia of the liver - mixed hyperplastic and adenomatous subtype}

Raul Guilherme Azevedo Macedo - TCBC-RJ'; Adriana Caroli-Bottino²; Vera lúcia Pannain³; Juliana Vial Paschoal; Vinicius

GOMES DA SILVEIRA - TCBC-RJ ${ }^{5}$

\section{INTRODUÇÃO}

\begin{abstract}
A hiperplasia nodular focal (HNF) acomete geralmente mulheres $^{1}$ e, embora seja pouco comum, é a lesão hepática benigna mais freqüente, depois do hemangioma ${ }^{2,3}$. Descrita por Edmondson em 19584, atualmente, a HNF é classificada morfologicamente em: clássica, a mais comum, e não-clássica. Esta última é ainda subdividida em 03 tipos: telangectásico, o mais freqüente ${ }^{2,5}$; misto (hiperplásico/ adenomatoso) e com atipias citológicas. Geralmente a HNF é uma lesão solitária, podendo ser múltipla em $20 \%$ dos casos $^{3}$. O diagnóstico diferencial com adenoma e o carcinoma hepatocelular é freqüente quando não se observa a clássica cicatriz central nos métodos de imagem. Relatamos a seguir um caso de HNF tipo misto (hiperplásico /adenomatoso).
\end{abstract}

\section{RELATO DO CASO}

Paciente de 51 anos, feminina, branca, natural do Rio de Janeiro, com desconforto em hipocôndrio direito há seis meses, sem relação com a alimentação e com piora no último mês. A ultrassonografia de abdome mostrou massa de $9 \mathrm{~cm}$ no lobo esquerdo do fígado, sendo encaminhada ao Hospital Universitário Clementino Fraga Filho da UFRJ. A avaliação laboratorial com marcadores tumorais (CEA, CA 19.9 e alfa-feto proteína), sorologia para vírus da Hepatite (A, B e C) e provas de função hepática foram normais. Foram evidenciadas quatro lesões nodulares sólidas e hipervasculares com lavagem moderada do contraste, a maior delas com $8 \mathrm{~cm}$, localizada em segmentos II/III do fígado (Figura 1). As hipóteses de metástase, carcinoma hepatocelular ou adenoma não foram afastadas.

Durante o ato operatório encontrou-se lesão de consistência firme, limites bem definidos localizadas no segmento III e três nódulos menores em segmentos VI e VII, todos com o mesmo aspecto da maior. O restante do in-

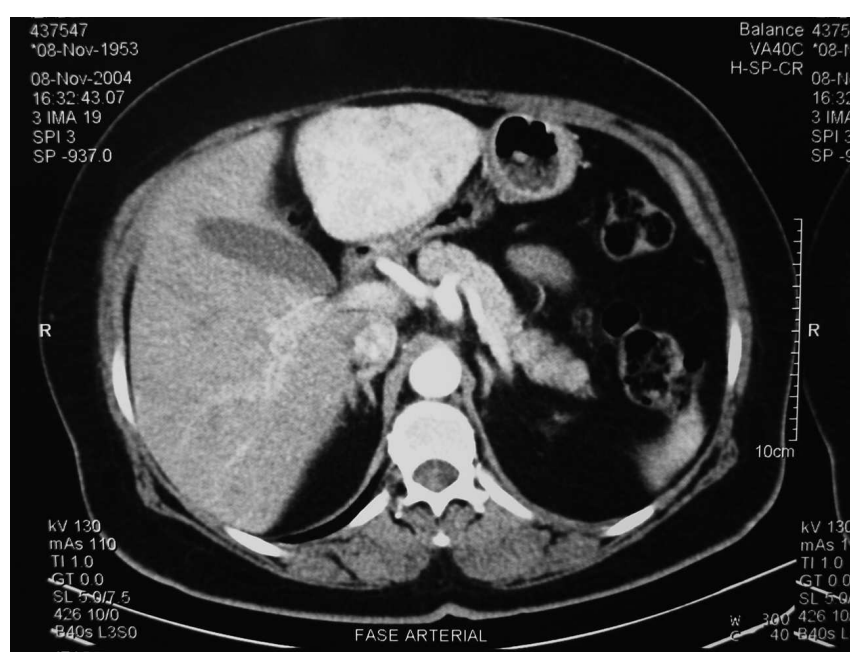

Figura 1 - Tomografia computadorizada de abdome mostrando a lesão maior, localizada em lobo esquerdo do fígado.

ventário da cavidade foi normal. Realizou-se segmentectomia do III, englobando toda a lesão maior. O exame per operatório de congelação afastou malignidade. A paciente apresentou boa evolução recebendo alta hospitalar no $3^{\circ}$ dia pós-operatório

O exame histopatológico evidenciou trabéculas de hepatócitos separadas por sinusóides, eventualmente duplas, além de fibrose focal e irregular tendo de permeio vasos e dúctulos biliares (Figura 2A e 2B). O diagnóstico foi hiperplasia nodular focal forma mista (hiperplásica/ adenomatosa).

\section{DISCUSSÃO}

A HNF é uma lesão cuja incidência vem aumentando nos últimos anos, talvez pela melhora nos métodos diagnósticos de imagem³ ${ }^{3}$.

Trabalho foi realizado no Hospital Universitário Clementino Fraga Filho - Universidade Federal do Rio de Janeiro - RJ-BR.

1. Professor Assistente de Cirurgia de Faculdade de Medicina de Petrópolis (FMP/FASE) - Petrópolis - RJ-BR; 2. Professora Adjunta do Departamento de Patologia da Faculdade de Medicina da Universidade Federal do Rio de Janeiro - RJ-BR; 3. Professora Adjunta do Departamento de Patologia da Faculdade de Medicina da Universidade Federal do Rio de Janeiro - RJ-BR; 4. Pós-Graduanda do Departamento de Patologia da Faculdade de Medicina da Universidade Federal do Rio de Janeiro - RJ-BR; 5. Professor Adjunto do Departamento de Cirurgia Geral da Faculdade de Medicina da Universidade Federal do Rio de Janeiro - RJ-BR. 


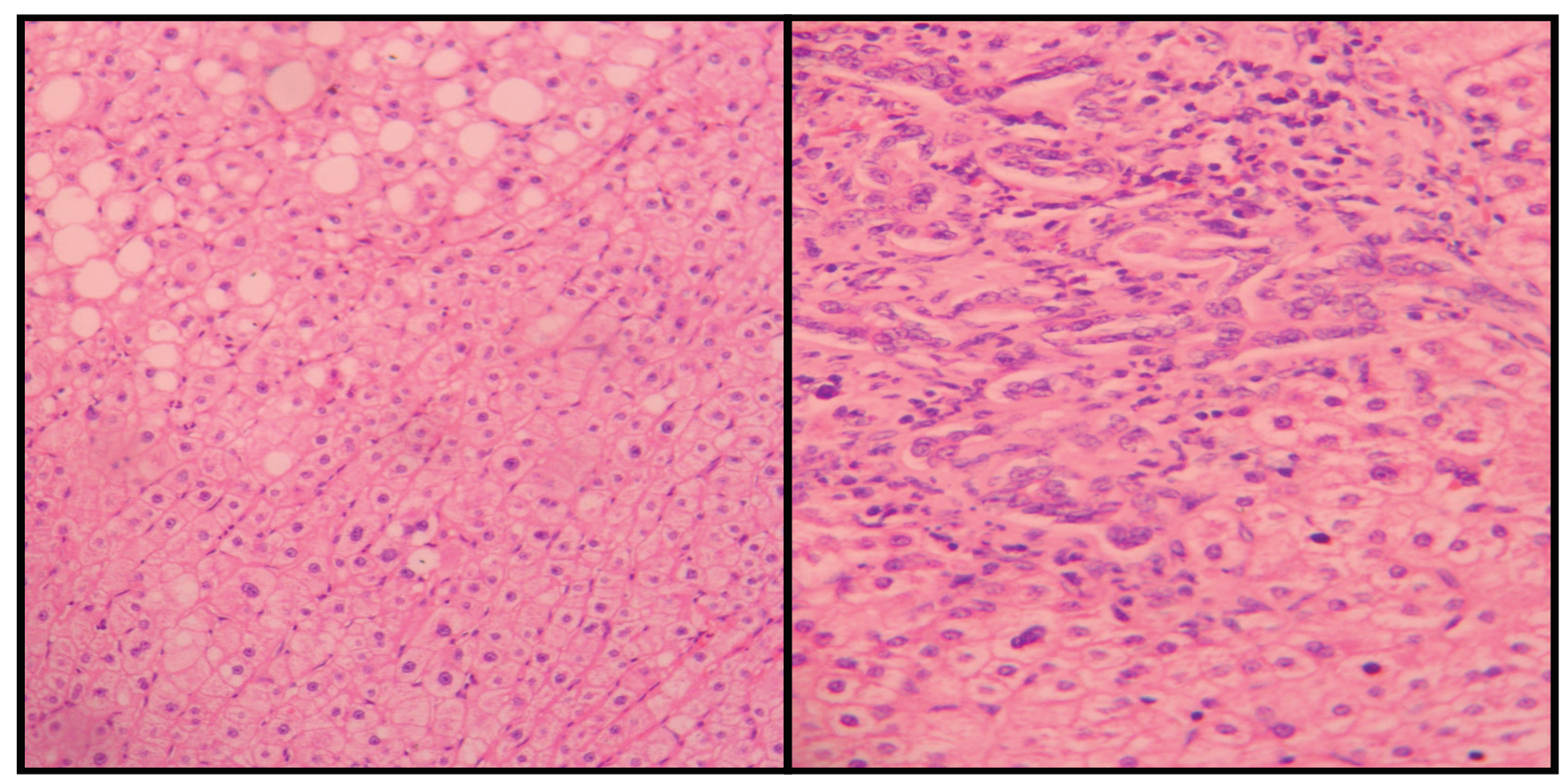

Figura 2 - A - Trabéculas hepatocitárias por vezes duplas delimitadas por sinusóides com presença de macrovacúolos lipídicos. B - Fibrose irregular permeada por dúctulos biliares proliferados.

Inicialmente, a conduta nos casos de HNF era quase sempre cirúrgica, pois não se conhecia bem o comportamento da lesão ao longo dos anos ${ }^{1}$. Entretanto, a abordagem se modificou a partir de uma melhor compreensão da sua fisiopatologia, que é baseada em uma malformação vascular e, principalmente, da sua história natural, de curso usualmente benigno.

A associação com o uso de contraceptivos orais (à semelhança dos adenomas) que foi inicialmente sugerida por alguns autores, não tem se mostrado significativa nas casuística publicadas ${ }^{1,3}$.

Atualmente, a maioria dos trabalhos advoga uma conduta expectante para a HNF, com controle anual por exame de imagem. A indicação cirúrgica ocorre quando o diagnóstico diferencial com adenomas ou tumores malignos não pode ser afastado, e/ou quando o paciente apresenta sintomatologia associada como dor incapacitante 2,3,5.

Nguyen e cols ${ }^{5}$ publicaram a maior casuística de HNF com 305 lesões ressecadas, sendo 80,3\% da forma clássica com a presença de cicatriz na maioria casos. Entre as formas não clássicas (19,7\%), apenas 5 lesões (1,6\%) foram do tipo misto hiperplásico/adenomatoso, o que, além do achado de lesões múltiplas, reforça a raridade do caso relatado.

Neste relato, a dúvida diagnóstica foi o fator determinante para a indicação da operação. Optamos por conduta cirúrgica mais conservadora com a ressecção apenas da lesão maior, baseados na evidência de que todas apresentavam o mesmo aspecto radiológico e cirúrgico, assim como no fato da biópsia per operatória ter afastado malignidade, pois a ressecção das demais implicaria em uma operação mais ampla que não traria benefícios para a paciente.

\section{A B S T R A C T}

Focal Nodular Hyperplasia is a benign lesion of the liver, which usually presents with one or two localizations. We report the uncommon case of a 51-year-old female who presents with right upper quadrant pain that worsened in the previous month, without association with feeding. Four hepatic lesions were evidenced at Computerized tomography, the largest being of $8 \mathrm{~cm}$ in diameter, of atypical behavior. She was submitted to hepatic segmentectomy of the segment III. The pathologic diagnosis returned focal nodular hyperplasia - mixed hyperplastic and adenomatous sub-type. The patient had a good postoperative evolution and is in ambulatory follow-up.

Key words: Focal nodular hyperplasia. Liver. Carcinoma hepatocellular. Diagnosis. Adenomatous polyps.

\section{REFERENCIAS}

1. D'halluin V, Vilgrain V, Pelletier G, Rocher L, Belghiti J, Erlinger S, et al. Natural history of focal nodular hyperplasia. A retrospective study of 44 cases. Gastroenterol Clin Biol 2001; 25(11):1008-10.
2. Hussain SM, Terkivatan T, Zondervan PE, Lanjouw E, de Rave S, ljzermans JN, de Man RA. Focal nodular hyperplasia: findings at state-of-the-art MR imaging, US, CT, and pathologic analysis. Radiographics 2004; 24(1):3-17; discussion 18-9. 
3. Chamberlain RS, DeCorato D, Jarnagin WR. Benign liver lesions. In: Blumgart LH, Fong $Y$, Jarnagin WR, editors. Hepatobiliary cancer Hamilton: BC Decker; 2001. p.1-30.

4. Edmondson HA. Tumors of the liver and intrahepatic bile ducts. In: Atlas of tumor pathology. Washington: Armed Forces Institute of Pathology; 1958. Parte inferior do formulário

5. Nguyen BN, Fléjou JF, Terris B, Belghiti J, Degott C. Focal nodular hyperplasia of the liver: a comprehensive pathologic study of 305 lesions and recognition of new histologic forms. Am J Surg Pathol 1999; 23(12):1441-54
Recebido em 25/02/2007

Aceito para publicação em 30/03/2007

Conflito de interesse: nenhum

Fonte de financiamento: nenhuma

\section{Como citar este artigo:}

Macedo RGA, Caroli-Bottino A, Pannain VL, Paschoal JV. Hiperplasia nodular focal múltipla - subtipo misto hipeplásico/adenomatoso. Rev Col Bras Cir. [periódico na Internet] 2011; 38(4). Disponível em URL: http://www.scielo.br/rcbc

Endereço para correspondência:

Raul Guilherme Azevedo Macedo

E-mail: macedoraul@ig.com.br 